Cinémas

Revue d'études cinématographiques

Journal of Film Studies

\title{
Document
}

\section{Georges Sadoul. Cours du samedi 22 janvier 1949}

Volume 19, numéro 2-3, printemps 2009

La filmologie, de nouveau

URI : https://id.erudit.org/iderudit/037556ar

DOI : https://doi.org/10.7202/037556ar

Aller au sommaire du numéro

Éditeur(s)

Cinémas

ISSN

1181-6945 (imprimé)

1705-6500 (numérique)

Découvrir la revue

Citer ce document

(2009). Document / Georges Sadoul. Cours du samedi 22 janvier 1949. Cinémas, 19(2-3), 269-285. https://doi.org/10.7202/037556ar d'utilisation que vous pouvez consulter en ligne.

https://apropos.erudit.org/fr/usagers/politique-dutilisation/ 


\section{Cours du samedi 22 janvier $1949^{1}$}

\section{Georges Sadoul}

Voir la note de la rédaction à la fin du document

Je voudrais aujourd'hui, où nous avons une séance de travaił, vous donner d'abord quelques renseignements bibliographiques, quelques renseignements aussi sur les possibilités qui yous seront ouvertes pour vos recherches, mais je voudrais strtout, comme nous disposons aujourd'hui d'un peu plys de temps, qu'un dialogue s'établît entre nous pour que yous me disiez dans quel sens certains d'entre vous qui conptent faire des recherches pensent les orienter, et, dans la limite de ma spécialité, j'essaierai de vous donner quelques indications sur les sources de documentation que vous polrriez trouver.

Comme je suis plus particulièrement historien du cinéma il est certain que les indications que je vais vous donner d'une façon géperale sont des indications qui vont beaucoup plus ported sur les travaux historiques, et accessoirement sur le langage du film.

quels sont les principaux genres de sources qu'on peut consulter? Sans chercher l'endroit où on peut les trouver, recherchons les différentes catégories de documents que vous pouvez être appelés à consulter[.]

Il y a d'abord les films eux-mêmes, ceci va de soi, mais il y a pour ceux-ci une grande difficulté, c'est la façon de réussir à consulter et à étudier véritablement de près ces films.

Si vous faites par exemple des travaux sur le langage du si vous voulez connaître les différentes études du langage du film dont je me suis efforcé dindiquer quelques-unes lors de mes précéden exposés, vous êtes naturellement appelés à étudier les 
films de près; mais pour étudier ces films, encore les consulter et un film ne pas avec la même facilité qu'un live.

Les films sont conservés dans le monde, dans des cinémathèques. Ces cinémathèques sont pour la plupart des organismes privés, ou semi-privés, pour les plus importantes d'entre elles tout au moins, largement subventionnées par l'Etat, mais ne disposant pas, à la différence des bibliothèques publiques, et en premier lieu de la Bibliothèque Nationale, des moyens qui sont ceux de la Bibliothèque Nationale; c'est-à-dire que tous les films ne sont pas conservés dans les cinémathèques et que les collections des cinémathèques sont généralement fort incomplètes.

D'autre part les cinémathèques, et je pense à la Cinémathèque française en particulier, qui est une des plus riches, et quoiqu'elle soit une des plus riches elle est de fondation récente, ne sont pas suffisamment équipées.

Si vous voulez étudier le langage du film et les classiques du cinéma vous avez actuellement un bon instrument de connaissance, je ne dis pas un instrument de travail, qui est en annexe de l'Exposition de la Cinémathèque: Les Cent Chefs d'œuvres $\mathrm{du}$ Cinéma, où vous pouvez voir cent à cent cinquante films fort importants.

Ceci ne veut pas dire que si vous voulez pousser plus loin vos études vous pouvez réellement les pousser, car il ne suffit pas de voir un film une fois pour le bien connaître, il fay pouvoir l'étudier, pouvoir se faire faire des projections individuelles, ou plus exactement des visions individuelles par les petits appareils qu'on appelle les moviolas, qui sont des appareils qu'on manie soi-même, dans lesquels on passe fle film et on a une petite image à peu près de la grandey d'une carte postale. On peut arrêter l'appareil, le faire allef en avant et en arrière, seulement je crois que l'Institut de Filmologie ne dispose pas encore de moviolas, et que d'autre part la Cinémathèque Française n'en dispose pas non plus, et lorsqu'on veut examiner un film on est obligé de Yexaminer purement et simplement à l'enrouleuse; c'est yn procédé d'un privitimisme extrême et évidemment fort incommode. 
Pour ce qui est du film lui-même il faut tout de même considérer qu'en dehors de la bande de celluloïd on peut trouver un certain nombre d'éléments qui permettent d'étudier davantage un film. Ces éléments sont pour les films anciens les publications qui ont pu être faites pour ces films; pour les films très anciens les catalogues contiennent une espèce d'esquisse du découpage qui permet d'avoir à peu près une idée du déroulement du film plan par plan. Pour les films les plus récents et pour les plus célèbres d'entre eux, fort heureusement, les découpages techniques, c'est-à-dire ces espèces de cahiers de 200 à 300 pages ronéotypées qui contiennent le plan de travail d'un film, ceci par numéros, chaque numéro correspondant à un plan du film, et un film comprend en moyenne 600 à 700 plans, ces découpages techniques contiennent d'un côté de la page le dialogue et de l'autre les indications de mise en scène, et ceci vous permet d'étudier a priori la conception du film telle qu'elle a été conçue par ses auteurs, telle qu'elle n'a d'ailleurs pas toujours été très exactement suivie dans le film, enfin ceci est quand même un très bon élément de recherches pour vous autour d'un film. Ceci vous permet de voir assez clairement ce qu'ont été les intentions des auteurs, intentions qu'ils ont ensuite réalisées ou non.

Les découpages des principaux films, français tout au moins, ont été déposés, soit à la bibliothèque de l'I.D.H.E.C., soit à la bibliothèque de la Cinémathèque. Seulement la bibliothèque de l'I.D.H.E.C. est actuellement fermée

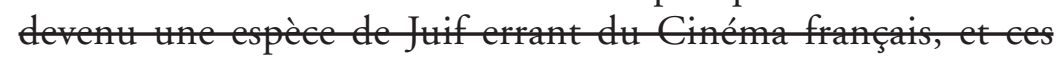
découpages sont actuellement dans des caisses, vous ne pourez Quant à la bibliothèque de la Cinémathèque, elle est ouverte de façon assez irrégulière, et je ne peux même pas vous indiquer si elle est ouverte à l'heure actuelle.

Ceci est fort regrettable parce que, j'en arrive maintenant à une autre source, la bibliothèque de la Cinémathèque Française est actuellement l'une des plus riches du monde. Vous y trouvez, dans la mesure où elle ouverte, dans la mesure où d'autre part il existe un fichier, parce que le fichier n'est pas encore terminé, il n'est pas à la disposition du public, vous y trouvez à peu près 
tous les livres importants qui ont été publiés, soit en France, soit à l'étranger, sur le Cinéma, et elle comporte je crois actuellement deux ou trois mille titres, ce qui est relativement considérable pour une bibliothèque. Il y a un certain nombre de livres très rares que vous ne pouvez trouver que là ; encore faut-il qu'elle soit ouverte.

Une autre source de bibliothèque que vous pouvez consulter, c'est la bibliothèque de l'I.D.H.E.C., que je suis obligé de citer pour mémoire puisque, actuellement, elle est fermée et absolument inaccessible, mais enfin il faut espérer qu'un jour pro

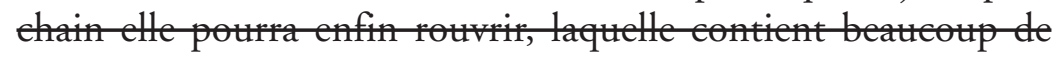
ehoses, mais elle est sensiblement moins riche que celle de la Ginémathèque.

Les buts ne sont d'ailleurs pas les mêmes. La bibliothèque de I'I.D.H.E.C. n'est pas par exemple une bibliothèque qui recherche systématiquement les livres rares; c'est une bibliothèque qui est avant tout un instrument de travail pour les étudiants, qui contient par conséquent surtout les livres courants. Elle a l'avantage de posséder d'autre part un fichier extrêmement important de films, qu'il est facile de consulter, dans la mesure, encore une fois, où cette bibliothèque est ouverte.

Vous avez d'autre part, pour ceux qui s'intéressent à la période de l'histoire du Cinéma antérieure à la dernière guerre, une bibliothèque qui est beaucoup plus riche que celle de la Cinémathèque, beaucoup plus riche que celle de l'I.D.H.E.C. pour ces périodes, qui est celle de l'Arsenal.

La bibliothèque de l'Arsenal $^{2}$, dont la Conservatrice est $\mathrm{M}^{\mathrm{me}}$ Ormonval, qui vous recevra fort aimablement si vous dites que vous venez de la part de l'Institut de Filmologie, et dont l'assistance vous sera nécessaire car, là non plus, il n'existe pas de catalogue et il faut se fier à la mémoire et à la bonne volonté, qui est d'ailleurs extrême, de la Conservatrice pour y trouver quelque chose.

La bibliothèque de l'Arsenal est une bibliothèque qui contient d'inappréciables ouvrages, pour la plupart assez anciens, et d'inappréciables collections de périodiques: toutes les revues de Cinéma publiées en France, et un certain nombre de revues publiées à l'étranger, surtout pour la période qui va de 


\section{Document}

1910 à 1930 environ. Elle a été constituée par un collectionneur qui s'appelait M. Rondel, elle appartient à ce qu'on appelle le «Fonds Rondel».

M. Rondel s'intéressait avant tout au Théâtre, et c'est seulement à la fin de sa carrière de collectionneur, qui est d'ailleurs très remarquable, qu'il a commencé à s'intéresser au Cinéma et qu'il a commencé à grouper un nombre considérable de documents, documents qui sont surtout des revues et des livres, mais dans lesquels vous pourrez trouvez des sources et des éléments extrêmement précieux.

Voilà à peu près pour ce que je connais de la France ce à quoi vous pourrez recourir. Ceux d'entre vous qui iraient à l'étranger pourraient consulter la bibliothèque de la British Film Institute, qui est une riche bibliothèque, sensiblement moins riche d'ailleurs que la bibliothèque française. Et d'autre part il existe à New-York une bibliothèque rattachée à la Cinémathèque [MoMA] qui paraît être aussi une bibliothèque assez riche; mais je ne connais pas... je connais assez bien celle de la British Film Institute, mais pas aussi bien la bibliothèque américaine. Pour ce qui est des autres pays les bibliothèques, et d'ailleurs les collections de films, sont assez insuffisantes.

Je voudrais maintenant passer en revue avec vous un certain nombre d'ouvrages qui se trouvent dans le commerce et qui pourront peut-être, pour certains d'entre eux, vous être utiles.

Je vais vous donner une bibliographie assez complète, et qui sera d'ailleurs une bibliographie critique. Je vais commencer par vous donner une bibliographie qui sera celle des ouvrages historiques publiés en français, ou dans des langues accessibles, telles que l'anglais, l'italien, enfin les langues qui sont généralement connues chez nous. Je vous citerai d'ailleurs un certain nombre de livres en vous disant de ne pas les consulter, simplement pour vous éviter l'achat de certains de ces livres dont les titres peuvent par exemple tromper. Alors je donne au hasard, comme cela vient, et à peu près par ordre alphabétique.

Il y a un livre de Pierre Artis, qui s'appelle "Histoire du Cinéma américain». Ne le consultez pas, ce n'est pas la peine d'en faire l'achat, ce n'est pas un livre intéressant; le titre est 
fallacieux, c'est simplement une revue des principaux films américains édités entre 1936 et 1940 en France, c'est une énumération de titres sans aucun point de vue critique, qui se contente de donner les noms des principales vedettes. Inutile donc de consulter ce livre.

J'en dirai autant d'un livre d'André Paul qui s'appelle «Le Cinéma et son Histoire", et qui ne parle ni du Cinéma ni de son Histoire, ou qui en tout cas n'en parle qu'avec aucune espèce d'efficacité.

Par contre, je recommande pour ceux d'entre vous qui s'intéressent - ceci n'étant d'ailleurs pas l'objet de la Filmologie, mais pouvant avoir certains effets sur la naissance du film et sa conception - je vous recommanderai par contre chaudement un livre de M. Pierre Baechlin, qui s'appelle "Histoire Économique du Cinéma", et qui a été publié à la Nouvelle Edition. Le livre de M. Baechlin est la traduction d'une thèse de doctorat en Droit soutenue devant l'Université de Bâle, en allemand. Le titre allemand est beaucoup plus exact, il veut dire «Le Film comme marchandise», et c'est en effet ce dont il s'agit, c'est beaucoup plus l'étude du film en tant que marchandise et tous les aspects économiques du Cinéma, [qu']une histoire économique du Cinéma, à proprement parler.

En tant qu'histoire économique du Cinéma, ce ne serait pas une bonne histoire économique du Cinéma, elle est assez incomplète, mais si on la considère sous cet angle, c'est-à-dire toutes les incidences que peut avoir le caractère de marchandise $\mathrm{du}$ film sur le caractère artistique et sur le caractère de moyen d'expression du film, son caractère de marchandise, cette étude est une bonne étude et vous pouvez vous y référer.

Il existe une "Histoire du Cinéma» de Coissac que je cite également pour mémoire. C'est un ouvrage déjà assez ancien paru en 1924, assez vieilli et qui ne touche à proprement parler que l'histoire de l'invention du Cinéma, c'est-à-dire ce qui pour vous, en somme, n'a pas grand intérêt. C'est un ouvrage qui a eu sa valeur, qui a eu son importance, mais qui ne me semble pas recommandable.

Je cite un ouvrage qui, par contre, pourra vous rendre un certain nombre de services, c'est le livre de Colpi, qui s'appelle 


\section{Document}

«Le Cinéma et ses hommes». Ce n'est pas à proprement parler un livre qui touche directement - il ne le touche d'ailleurs nullement - le langage du film, c'est, si vous voulez, une espèce de petit dictionnaire où vous avez l'avantage de trouver les principaux noms des metteurs en scène dans les différents pays et leurs principales œuvres.

C'est rempli d'erreurs, il y a toutes sortes d'erreurs de dates en particulier, quelquefois même quelques petites erreurs d'attribution, mais les erreurs sont toujours inévitables dans des travaux de ce genre et il ne faut pas y attacher trop d'importance.

La conception de ce livre, d'autre part, se limite aux films compris entre 1930 et 1945 . C'est cependant un ouvrage qui pourra vous être assez utile, tout au moins pour ceux d'entre vous qui s'intéressent aux travaux historiques. C'est commode comme un dictionnaire, un dictionnaire qui n'est pas toujours très bien fait, mais c'est commode comme un dictionnaire et c'est vraiment assez agréable, assez utile de l'avoir sous la main pour faire certains travaux de recherches.

J'en viens maintenant à un livre qui est l'un des plus célèbres dans l'Histoire du Cinéma, qui est le livre de Bardèche et Bra[sillach], qui s'appelle "Histoire du Cinéma», dont il vient d'avoir récemment une réédition.

C'est un livre qui a eu incontestablement son utilité au moment où il a été écrit, malgré les erreurs ou le parti pris de certains points de vue, mais dont la valeur historique est véritablement extrêmement contestable. Vous y trouverez d'ailleurs assez peu de considérations sur, par exemple[,] le langage du film, et pouvant vous servir par exemple pour la Filmologie.

Sa première édition avait l'avantage d'être un livre d'une lecture extrêmement courante, extrêmement facile; cela se lisait comme un roman. L'inconvénient c'est que la plupart des dates étaient fausses et que même très souvent les attributions des films l'étaient également; on attribuait à un metteur en scène des films qui n'avaient jamais été de lui, et évidemment du point de vue historique c'est assez regrettable et assez criti[qu]able.

Les éditions suivantes, et surtout la dernière, ont été gâchées, non seulement par un parti pris beaucoup plus accentué que dans les précédentes éditions, mais par des additions successives 
qui ont rendu ce livre, qui était autrefois d'une lecture entraînante, assez difficile à suivre et souvent assez ennuyeux, surtout dans la dernière édition, paraît-il.

Ces critiques faites il faut reconnaître que ce livre a l'avantage d'avoir un index, d'être facilement consultable et quelquefois d'être assez commode.

"Le Panorama du Cinéma», de Char[e]nsol, qui a été réédité récemment, a été en 1928 un excellent ouvrage, le premier travail d'ensemble qui ait été fait sur l'ensemble du cinéma. Malheureusement l'ouvrage a vieilli et l'édition revue et corrigée récemment n'a pas été suffisamment, à mon avis, revue et corrigée. Je ne crois pas que ce soit un ouvrage dont vous aurez beaucoup à vous servir.

Par contre voici deux excellents livres que je vous recommande chaleureusement, et qui n'ont qu'un inconvénient, c'est de ne pas être français.

Il y a d'abord l'ouvrage de Lewis Jaco[b]s, dont le titre veut dire "La croissance, le développement du film américain" [The Rise of the American Film. A Critical History, 1939].

Comme son titre l'indique cet ouvrage, qui est l'un des meilleurs existant en tant qu'histoire du Cinéma s'intéresse seulement au Cinéma américain, mais avec des vues assez larges pour recourir et pour indiquer en quelques pages l'essentiel de l'apport des écoles étrangères qui ont eu de l'influence et une incidence sur le Cinéma américain.

Le plan du livre est assez systématique, un peu trop systématique peut-être, c'est-à-dire qu'il est généralement composé pour chacune de ses parties en trois chapitres: l'un étudiant les aspects économiques du problème, l'autre ses aspects artistiques à travers les noms des principaux réalisateurs, et pour terminer leurs aspects sociaux, ceci à travers une analyse des titres de films.

Ce plan est peut-être quelquefois un peu rigide et un peu systématique, mais, répétons-le, ce livre est un des meilleurs, peut-être le meilleur en tant qu'histoire du Cinéma, qui ait été publié à travers le monde.

Un autre livre, en anglais également, est un livre de Kracauer. C'est un livre en anglais, mais écrit par un Allemand, et qui s'appelle "De [Caligari] à Hitler ", "From [Caligari] to Hitler ". 


\section{Document}

C'est une étude consacrée uniquement au cinéma allemand, et au cinéma allemand pour cette courte période, mais fort riche, qui va de 1920 à 1933 .

Il s'agit pour une part d'une étude du langage du film, mais surtout d'une étude du sujet des films, des thèmes sociaux à travers le cinéma allemand durant cette période. C'est un ouvrage peut-être un peu systématique; on a l'impression à quelques reprises que Kracauer sollicite quelquefois un petit peu le sujet des films dans un sens qui est celui de ses thèses, mais néanmoins Cc'est un ouvrage extrêmement précieux, extrêmement intéressant et irremplaçable en ce qui concerne cette période très riche qu'est cette période du cinéma allemand.

J'en viens maintenant à deux anthologies qui pourront vous être utiles, l'une, celle de [Marcel] Lapierre, qui s'appelle "Anthologie du Cinéma", et l'autre celle de Marcel [LH] erbier, qui s'appelle "Intelligence du Cinéma[tographe]", dans lesquelles vous trouverez non seulement des textes historiques, mais des textes esthétiques, et souvent des textes assez précieux, car il vous sera difficile de les trouver ailleurs. Ce sont des textes extraits de revues, extraits de livres épuisés, et textes qui ne sont pas toujours très faciles à consulter et à retrouver même.

Ces deux livres ont l'avantage de ne jamais faire double emploi et d'être au contraire complémentaires. L'anthologie de Lapierre est orientée dans un sens plus particulièrement historique, pour certaines de ses parties tout au moins; le livre de [LH] erbier est orienté dans un sens esthétique, et il vous sera plus particulièrement précieux, je pense, en ce qui concerne les théories esthétiques qui sont celles de l'époque de [L'H]erbier, de l'époque où [L'H] erbier a fait les débuts de sa carrière, ce sont les théories esthétiques françaises ayant cours en France entre 1920 et 1930, pendant l'espèce d'âge d'or du cinéma muet.

D'ailleurs, dans le livre de Lapierre vous trouverez également une série de textes fort intéressants, et par exemple certains textes écrits — je prends un exemple au hasard — par Marcel Carné à l'époque où il était journaliste, et qui constituent un petit peu une clé de l'œuvre de Marcel Carné.

Lapierre vient également de publier aux Éditions Grasset un énorme ouvrage qui s'appelle «Les [cent] visages du Cinéma». C'est 
un livre fort épais, fort coûteux d'ailleurs, qui est un ouvrage fort riche en documentation. Il faut néanmoins regretter deux choses: c'est que cette documentation soit, d'une part présentée toujours sous un aspect peut-être un peu brut, ce qui rend quelquefois l'étude du livre un peu difficile, et que d'autre part, pour la recherche et pour le travail - et encore une fois cet ouvrage contient une documentation fort intelligemment choisie et par certains côtés inappréciable - il n'existe pas d'index à ce livre, et les recherches y sont véritablement fort difficiles. C'est un livre qu'il faudrait presque ficher pour réussir ensuite à travailler sur lui.

Pendant que j'en suis à ces livres sur le Cinéma je vous signale un autre livre d'histoire du Cinéma, qui est fort commode celuici, et qu'il est fort difficile de trouver, c'est un ouvrage en italien de Pasinetti, qui s'appelle ["Storia del Cinema dalle origini a oggi»].

Pour ceux d'entre vous qui lisent l'italien c'est un livre commode. Ce n'est pas un livre qui vous dise grand'chose sur la substance des films, mais un livre qui vous rendrait les mêmes services que le Colpi, c'est-à-dire qu'ayant à faire un travail historique sur un film, voulant connaître à peu près ses tenants, ses aboutissants, sa date de fabrication, ses principaux auteurs et ses acteurs vous pourrez consulter le livre de Pasinetti avec la même sûreté qu'on consulte un annuaire du téléphone. Les points de vue critiques n'y sont pas toujours très développés, mais, comme ouvrage de travail, c'est un ouvrage excellent et extrêmement utile. Il a cet inconvénient de s'arrêter à 1939, parce que c'est un livre de 1939 et qui n'a pas été mis à jour depuis.

A défaut de ce livre vous pouvez trouver une espèce de condensé de ce livre, qui se trouve facilement dans le commerce, du même auteur, qui s'appelle "Mezzo secolo de Cinema ", "Un demi-siècle de Cinéma», de Franc[es]co Pasinetti également. Ce livre, je l'ai, mais je consulte toujours l'autre, je n'ai pas de grandes vues sur la valeur exacte de ce résumé.

Je vous cite pour mémoire certains ouvrages, soit impossibles à se procurer, soit très difficiles à lire, du moins je le pense, pour la plupart d'entre vous.

Un ouvrage de [Terry] Ramsaye, qui est un ouvrage américain classique [A Million and One Nights], mais dont je crois 
qu'il n'existe que quelques exemplaires en France et aucun dans les bibliothèques publiques.

Je cite également un livre qui me paraît excellent à toutes sortes d'égards de M. Smrs, mais qui a l'inconvénient d'être en tchèque, et la lecture du tchèque est, je pense, assez impossible pour la plupart d'entre nous.

C'est un livre dont j'ai regardé fort attentivement les images et, en m'aidant de dictionnaires, j'ai réussi à comprendre quelques phrases, très imparfaitement du reste, pour la première partie, et il contient pour la seconde partie - et alors ça je n'y comprends rien du tout — des considérations qui me paraissent être sur l'esthétique du Cinéma. En tout cas je connais M. Smrs, je sais que c'est un homme extrêmement sérieux et qui fait d'excellent travail.

Ce livre est à la bibliothèque de la Cinémathèque; si vous avez quelques lueurs de tchèque consultez-le et vous verrez ce que vous pourrez en tirer. Malheureusement, pour moi personnellement je puis vous dire seulement que c'est un ouvrage extrêmement sérieux, et c'est tout.

Un livre qui peut vous rendre des services, mais qui je crois, maintenant, est assez difficile à trouver, c'est le livre de Carl Vincent qui s'appelle "Histoire de l'Art cinématographique", qui a l'avantage d'être abondamment et très bien illustré, mais qui contient beaucoup d'erreurs. C'est un livre déjà ancien, qui a vieilli d'ailleurs par beaucoup de côtés, mais qui, en particulier en ce qui touche le film allemand, contient de très bons chapitres.

Je signale en passant deux ouvrages qui peuvent vous être utiles, précisément par leurs illustrations: un ouvrage qu'on trouve assez facilement à Paris, de T[im] Taylor. C'est un ouvrage qui contient des renseignements sur les films américains ayant une valeur esthétique depuis cinquante ans. Et dans le même ordre d'idées, beaucoup mieux fait, avec beaucoup plus de goût, le livre de [Védrès] qui s'appelle "[Images] du Cinéma Français ", qui vous permet d'apprécier l'esthétique de certains films.

J'en ai à peu près terminé avec cette revue des livres. Je me permets de signaler les ouvrages que j'ai publiés moi-même, le 
premier volume de mon «Histoire Générale du Cinéma» que j'ai consacré à l'invention du Cinéma proprement dite et où vous ne verrez véritablement, je pense, aucune chose qui puisse directement vous intéresser, parce que c'est l'histoire des inventions des appareils, avec des considérations économiques diverses, mais où le langage et l'esthétique du film n'apparaissent pas encore puisque, aussi bien, mon livre s'arrête à 1897, c'està-dire une période où le film tel que nous l'entendons au sens moderne n'est pas encore né; il n'existe encore que des photographies animées, rien de plus.

Par contre, je crois que vous pourrez trouver certains éléments, d'information tout au moins, dans mon deuxième volume qui s'appelle "Les Pionniers du cinéma", qui va jusqu'en 1909, et où il y a une étude assez poussée de la formation du langage cinématographique, aussi bien à propos de Méliès que de ce que j'ai appelé [l'école de Brighton] et les premiers essais de L... ${ }^{3}$

J'en ai terminé maintenant avec l'Histoire du Cinéma proprement dite. Je voudrais vous signaler sur l'art du film, le langage $\mathrm{du}$ film, un certain nombre d'ouvrages, d'ailleurs la liste sera extrêmement restreinte.

D'ailleurs la plupart de ces ouvrages sont extrêmement difficiles à se procurer en France, et ce sont des ouvrages qui sont pour la plupart en langue anglaise ou qui n'existent qu'en traduction anglaise, ou exceptionnellement en traduction italienne.

Tout d'abord il y a un ouvrage que, je crois, vous aurez grand intérêt à consulter pour faire le point de certains problèmes du Cinéma, entre 1920 et 1930 tout spécialement, c'est l'ouvrage de M[oussinac], qui s'appelle «L'Age ingrat du Cinéma», publié aux Éditions du Sagittaire.

"L'Age ingrat du Cinéma» est un recueil d'articles, une espèce de somme de l'œuvre d'un homme qui a été le fondateur de la critique cinématographique en France, et où vous trouverez du point de vues historique, du point de vue de la conception que l'on avait du Cinéma et du film dans les 
différentes périodes, une étude extrêmement précieuse, en particulier celle qui ouvre le livre et qui a été publiée autrefois par Le Mercure de France.

Pour citer un nom qui est assez inséparable de celui de M[oussinac], c'est celui de Louis Delluc, mort depuis longtemps. Ceux d'entre vous qui s'intéressent aux conceptions qu'on pouvait avoir du langage du film et du rôle du cinéma aux environs de 1920 gagneront à consulter les ouvrages de Louis Delluc, et en premier lieu le livre qui s'appelle «Photogénie», et également "Cinéma et Compagnie». Ce sont des livres d'ailleurs absolument introuvables et que je n'ai pas vus, pour ma part, depuis dix ans chez les libraires, mais ils existent tout au moins dans les bibliothèques publiques, la bibliothèque de l'Arsenal en particulier.

Il y a parmi les publications anglaises récentes un excellent petit livre, qui est je crois assez facile à se procurer à Paris et que je vous recommande assez chaleureusement car il pose très bien les problèmes du Cinéma, de [Ernest Lindgren], [Lindgren] étant un des conservateurs de la Cinémathèque anglaise, et le titre est "L'Art du Film» [ "The Art of Film. An Introduction to Film Appreciation»]. C'est une étude de tous les aspects du Cinéma et une très bonne monographie sur la façon dont on opère le découpage d'un film dont on obtient certains effets, avec des considérations esthétiques et sur le langage qui sont fort intéressantes.

Maintenant il y a enfin, sur le découpage et sur le langage du film, deux ouvrages classiques, qui n'ont qu'un inconvénient, c'est de n'avoir été traduits en français ni l'un ni l'autre. C'est le livre d'Eisenstein et de celui de Poudovkin[e], «Le sens du film » [«The Film Sense»] pour le premier et l'autre porte, tout au moins dans sa traduction anglaise, le titre de «L'Art du film».

"Le Sens du film»d'[Eisenstein] — que, je dois dire, je connais fort mal — vient d'être réédité en anglais récemment. Il est donc possible pour ceux d'entre vous qui connaissent la langue anglaise de vous le procurer.

Une édition des ouvres complètes d'[Eisenstein], revue par Eisenstein lui-même, en français était en cours de préparation l'an dernier. Il devait y avoir cinq volumes, malheureusement la 
maison d'édition [Éditions Jacques Melot] a fait faillite et cette publication a été rejetée aux calendes.

Le livre de Poudovkin[e], «L'Art du film» est lui, par contre, absolument introuvable, même dans sa traduction anglaise. Fort heureusement il y a deux livres de Poudovkin[e], il y en a un sur "L'Art du film», qui est celui qui vous intéresserait davantage, mais il y en a un autre sur l'Art de l'Acteur, sur l'emploi de l'acteur dans le Cinéma [«Film Technique and Film Acting»]. Ce dernier, dont je ne me rappelle pas exactement le titre, vient heureusement d'être traduit en italien, et ceux d'entre vous qui lisent l'italien pourront se le procurer aux éditions de la revue [Bianco e Nero], qui est celle de l'Institut du Film, [Centre] expérimental du Cinéma à Rome.

Il existe également, je crois, une traduction italienne des deux livres d'Eisenstein et Poudovkin[e], mais déjà épuisés et assez difficile à se procurer.

Voici un tour d'horizon, que je sais moi-même extrêmement sommaire et incomplet, et j'ai d'ailleurs l'impression de ne pas vous avoir fourni tous les renseignements que vous désireriez parce que, lorsque j'ai regardé ma bibliothèque ou mes fiches, je me suis trouvé devant cette question que je n'ai pas pu résoudre: Qu'est-ce qui va exactement intéresser les gens avec qui je travaille?

C'est pourquoi je voudrais vous demander maintenant d'établir un dialogue, de me poser quelques questions. J'espère qu'à certaines d'entre elles, si vous me dites: Moi je m'intéresse plus particulièrement à ceci ou à cela, $j$ 'espère pour certaines de vos questions pouvoir vous dire: Bon, pour ça vous allez procéder de telle ou telle façon et je vous recommande tel ou tel ouvrage. Pour les questions auxquelles je ne pourrai pas répondre immédiatement, je prendrai vos demandes par écrit, j’y réfléchirai, et, dans une autre séance, je vous donnerai une réponse, qui pourra être utile je pense d'ailleurs, non seulement pour la personne qui m'aura posé la question, mais pour ceux d'entre vous qui s'intéresseraient à des questions connexes. 
Alors, je voudrais maintenant que le dialogue s'établisse entre nous. Je vous répète, je ne vous ai donné véritablement qu'une vue d'ensemble extrêmement fragmentaire et extrêmement incomplète.

Je voudrais cependant insister sur un dernier aspect de la difficulté de la recherche filmographique et de la recherche filmologique en général; en particulier en ce qui touche les périodes primitives, la naissance du langage, les recherches se trouvent parfois être d'une difficulté extrême. Je parle actuellement à la fois des sources sur celluloïd et des sources sur papier.

En ce qui touche les sources sur celluloïd, je prends actuellement la période primitive, je vous donne les difficultés pour cette période en vous soulignant néanmoins que ces difficultés sont beaucoup moins grandes lorsqu'il s'agit de périodes contemporaines. Mais lorsqu'on étudie le problème des origines dans les films on se heurte à des difficultés immenses et pratiquement insurmontables, ce qui fait que d'ailleurs les conclusions de certains travaux, des miens par exemple, ne peuvent être considérées que comme des hypothèses provisoires, des hypothèses de travail.

Alors, pour ce qui est des sources sur celluloïd il faut poser en règle que la quasi totalité des films antérieurs à la guerre de 1914 ont été détruits. On n'en parle plus; il reste peut-être un film sur cent ou un film sur mille. Fort heureusement les négatifs existent dans des proportions beaucoup plus fortes. C'est-à-dire que, pour prendre un exemple en France, la totalité des archives de la Maison Lumière est conservée par la Cinémathèque française; une grande partie des archives de Pathé et de Gaumont qui sont les principales maisons françaises, ont également été conservées, et, comme d'autre part Pathé et Gaumont avaient des succursales dans toute l'Europe, le fait que les archives de Pathé et Gaumont existent permet de connaître le développement du Cinéma en Russie, en Tchécoslovaquie, en Allemagne ou en Suède. Ces archives n'ont qu'un inconvénient, c'est qu'elles sont en négatifs, que, étant en négatifs, pour pouvoir les consulter il faut pouvoir les tirer en positifs, et que ceci suppose des crédits énormes, dont évidemment un chercheur particulier ne saurait disposer, car il s'agit de millions de 
francs, dont aucun institut d'Etat, c'est-à-dire, soit la Cinémathèque, soit l'I.D.H.E.C., ne dispose davantage. Donc, ces sources existent, mais elles n'ont qu'un inconvénient: elles sont absolument inconsultables.

On en est donc réduit aux films tels qu'ils existent, tels qu'ils ont été conservés absolument au hasard. Il se trouve que, quelque part en France, un forain a gardé ça dans un coin et que ceci est arrivé par quelque voie ou moyen dans les réserves de la Cinémathèque française.

Allons-nous en déduire que tel ou tel film a été à l'origine de telle ou telle expression de langage cinématographique? Ceci est extrêmement difficile à définir et extrêmement douteux. Nous sommes donc obligés de ne pas nous fier aux films uniquement, mais de nous reporter aux analyses des films qui ont pu être données par les catalogues de cette époque. Mais là, nous nous heurtons à une autre difficulté. Les catalogues des films français étaient des prospectus commerciaux; la Bibliothèque Nationale ne les a par conséquent pas conservés. Et d'ailleurs, si vous voulez faire des recherches de Cinéma à la Bibliothèque Nationale je vous dis à l'avance qu'elles sont à peu près impossibles. Il est extrêmement difficile de se débrouiller dans un fichier extrêmement complexe, assez mal tenu en ce qui concerne d'ailleurs le Cinéma, et d'autre part, non seulement les catalogues n'ont pas été conservés, mais toutes les publications, et elles étaient déjà nombreuses en France, qui existaient avant 1914 n'ont même pas fait l'objet de dépôt légal; c'est-à-dire que même ces collections de publications on ne peut pas les trouver à la Nationale.

En somme, pour les catalogues mêmes, nous en sommes à peu près réduits à la même chose que pour les films. Néanmoins, pour les titres et les analyses de films que nous connaissons, le nombre est infiniment plus grand, mais nous savons que, là aussi, les choses sont extrêmement incomplètes, et si, comme je vous l'expliquais dans l'exposé historique que je vous ai fait, certains d'entre vous s'intéressaient par exemple à l'origine du montage ou de la cinématographie, ou à l'origine de telle ou telle chose de langage, si certains d'entre vous s'intéressaient à des problèmes historiques, encore une fois vous vous trouverez devant d'extrêmes difficultés de recherches. 
Enfin je répète, pour les périodes récentes — et les périodes récentes vous intéressent bien davantage, c'est l'effet du cinéma sur le public contemporain qui est le principal objet de certaines de vos recherches, j'en suis certain d'avance - encore une fois le travail vous sera infiniment plus facile.

Maintenant, cette conclusion ou cette parenthèse faite, je voudrais vraiment que vous commenciez à me poser des questions et qu'un dialogue s'établisse entre vous et moi.

\section{Note de la rédaction}

Le document qui précède a été établi à partir du fac-similé de l'original déposé en archives. Le texte source est la transcription d'une intervention orale qui n'aurait été que partiellement révisée par l'auteur. Les biffures reproduisent celles qui apparaissent sur l'original et elles sont vraisemblablement de la main de Sadoul. Afin de nous conformer autant que possible au texte source, nous n'avons pas soumis ce document au protocole de la revue ni au processus de révision de la langue. Toute modification par rapport au fac-similé apparaît entre crochets.

\section{NOTES}

1. Cinémathèque française, fonds Georges Sadoul, GSA 103.1, selon les recherches de Valérie Vignaux.

2. Souligné à la main dans l'original.

3. Il s'agit vraisemblablement des premiers essais de Lumière. 